Check for updates

Cite this: RSC Adv., 2018, 8, 34408

\title{
Genome-wide identification and characterization of long non-coding RNAs responsive to Dickeya zeae in rice $\uparrow$
}

\author{
Wen Qi Li, (D) abc Yu Lin Jia, ${ }^{d}$ Feng Quan Liu, ${ }^{e}$ Fang Quan Wang, ${ }^{\text {ab }}$ Fang Jun Fan, ${ }^{\text {ab }}$ \\ Jun Wang, ${ }^{\mathrm{ab}}$ Jin Yan Zhu, ${ }^{\mathrm{ab}}$ Yang $\mathrm{Xu}^{\text {ab }}$ Wei Gong Zhong ${ }^{\mathrm{ab}}$ and Jie Yang ${ }^{\star a b}$
}

Plant long non-coding RNA (IncRNA) is a type of newly emerging epigenetic regulator playing a critical role in plant growth, development, and biotic stress responses. However, it is unknown whether IncRNAs are involved in resistance responses between rice and Dickeya zeae, a bacterial agent causing rice foot rot disease. In this study, RNA-seq was performed to uncover the co-expression regulating networks mediated by $D$. zeae responsive IncRNAs and their candidate target genes. Of the 4709 IncRNAs identified, 2518 and 2191 were up- and down-regulated in response to $D$. zeae infection, respectively. Expression changes of 17 selected IncRNAs and their predicted targets with a potential role in defense response were investigated by qPCR. The expression levels of five IncRNAs were up-regulated while their cognate candidate target genes were down-regulated upon D. zeae infection. In addition, several IncRNAs were predicted to be target mimics of osa-miR396 and osa-miR156. These results suggest that IncRNAs might play a role in response to $D$. zeae infection by regulating the transcript levels of their targets and miRNAs in rice.

Received 11th June 2018

Accepted 9th September 2018

DOI: $10.1039 / \mathrm{c} 8 \mathrm{ra04993a}$

rsc.li/rsc-advances an important role in its pathogenicity. ${ }^{3}$ Cultivating and planting resistant rice varieties against $D$. zeae is one of the most economical and effective methods for controlling this bacterial disease. However, the underlying genetic mechanism resistant to $D$. zeae is unknown in rice.

Similar to other plant species, rice has evolved a sophisticated defense mechanism to protect itself from constant invasions of pathogenic microbes. ${ }^{4}$ Currently, increasingly studies discovered that lncRNAs play a role in resistance of a diverse of fungal and bacterial diseases in rice., ${ }^{5,6}$ Based on the genome location, lncRNA are divided into three categories: (1) long intergenic non-coding RNAs (lincRNAs), (2) natural antisense transcripts (NATs) and (3) intronic lncRNAs. ${ }^{7}$

Coupled with the fast development of genome-wide transcriptome sequencing technology, a large amount of lncRNAs responsive to biotic and abiotic stresses have been identified in plants. ${ }^{89}$ Recently, a great number of differentially expressed IncRNAs were detected in rice and Arabidopsis after infection with the fungal pathogens, Magnaporthe oryzae and Fusarium oxysporum, respectively. ${ }^{10,11}$ Moreover, deep sequencing profiling uncovered a large number of lncRNAs in rice. ${ }^{12,13}$ The biological functions of most lncRNAs are still unknown although a few lncRNAs were shown to play a role in plant growth and development. For example, the expression level of a male-fertility-associated RNA (IncRNA LDMAR) was inhibited under long day conditions. Reduction of LDMAR transcript resulted in programmed cell death leading to male sterility due to failed anther development. ${ }^{14}$ Another rice lncRNA 


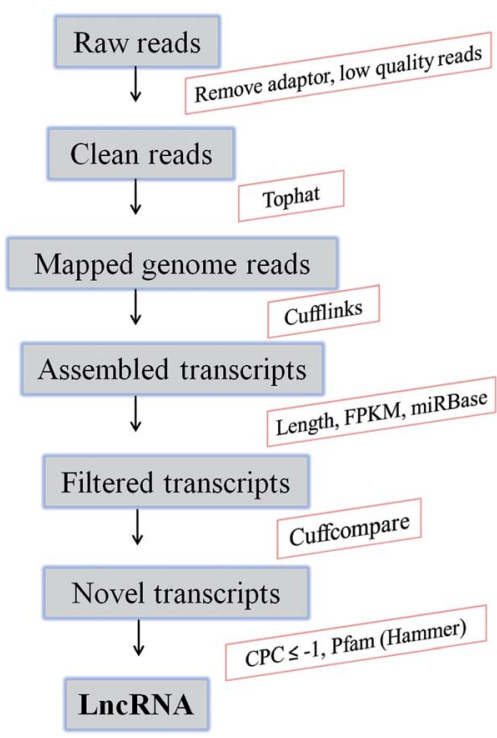

Fig. 1 The computational pipeline for identification of IncRNAs from RNA-seq data in resistant rice. CPC, Coding Potential Calculator.

XLOC_057324 has been shown to play a role in panicle development and sexual reproduction. ${ }^{15}$

Competing endogenous RNAs (ceRNAs) of miRNAs or miRNA target mimics have been shown to function in many biological processes through repression of miRNAs. ${ }^{16,17}$ Plant ceRNAs can act as 'sponges' to sequester miRNAs complementary to ceRNAs resulting in unleashing miRNA targets mimics. ${ }^{18,19}$ For instance, rice lncRNA XLOC_057324 is able to release more transcripts of the targets of miR160 and miR164 by decoying these two miRNAs. ${ }^{15}$ A ceRNA network was constructed for rice root and shoot tissues under phosphate starvation conditions. ${ }^{20}$ However, it is unclear whether IncRNAmiRNAs-target interaction networks are involved in immune response to pathogenic bacteria $D$. zeae in rice.

Studies on the role of IncRNAs in resistance responses can benefit to understand the rice immune mechanism. The objectives of the present study were to (1) identify and characterize lncRNAs in response to inoculation of D. zeae in a resistant rice variety, and (2) explore the crosstalk network among lncRNAs, miRNAs and their targets.

\section{Materials and methods}

\section{Plant materials and growth conditions}

All rice plants including Nanjing 40, Nanjing 45, Kasalath and Nipponbare were planted in experimental field under natural normal growth conditions, greenhouse and illumination incubator for bacterial pathogen D. zeae inoculation and RNA extraction in Jiangsu Academy of Agricultural Sciences, Nanjing, Jiangsu Province, China.

\section{Pathogen inoculation}

The resistance of rice plants to rice foot rot disease pathogen $D$. zeae was measured by the basal stem and root inoculation method. The resistance rate was classed as five groups by measuring the percentage of diseased area at 10 days after inoculation. No obvious disease symptom in the stem and foot indicates immunity. The disease index less than or equal to 5 indicates highly resistance. A disease index from 5.1 to 12.4 indicates moderately resistance. A disease index from 12.5 to 19.9 indicates moderately susceptibility. A disease index equals to or greater than 20 indicating highly susceptibility. ${ }^{21}$

\section{Library construction and sequencing}

The resistant japonica rice variety Nanjing 40 was selected for IncRNA library construction and RNA sequencing. Trizol kit (TaKaRa, Dalian, China) was used to extract root total RNAs from two samples, K1 (0 hour post inoculation, hpi) and K2, a mixed sample of 6,12 and 48 hpi based on three time biological replicates. ${ }^{22}$ The high-throughput RNA sequencing was performed at the LC-Bio Co., Ltd (Hangzhou, China) following the vendor's recommended protocol. The RNA quality was examined with an absorbance at $260 / 280 \mathrm{~nm}$ by using an Agilent 2100 Bioanalyzer. Approximately $5 \mu \mathrm{g}$ of total RNA was used to

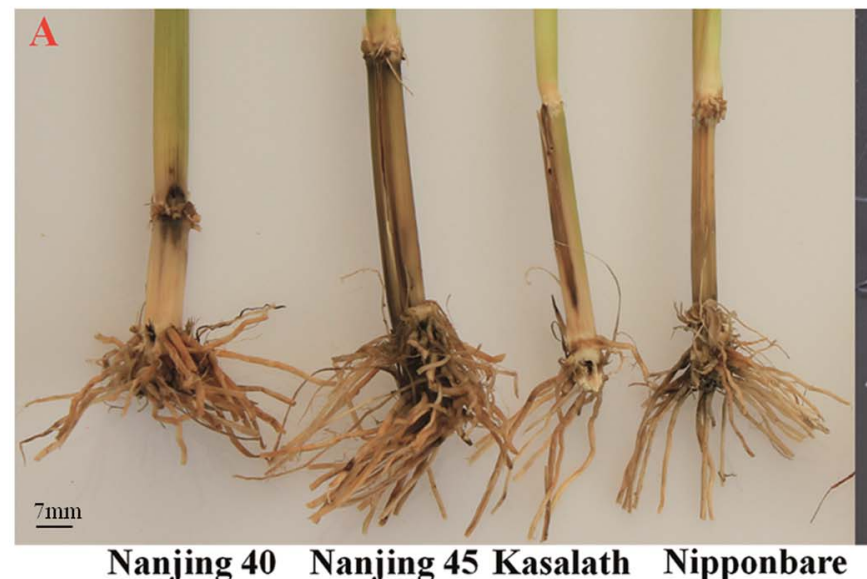

Fig. 2 Resistant and susceptible phenotypes in response to D. zeae infection. (A) and (B) phenotypes observed based on the method of basal stem and root inoculation, respectively.

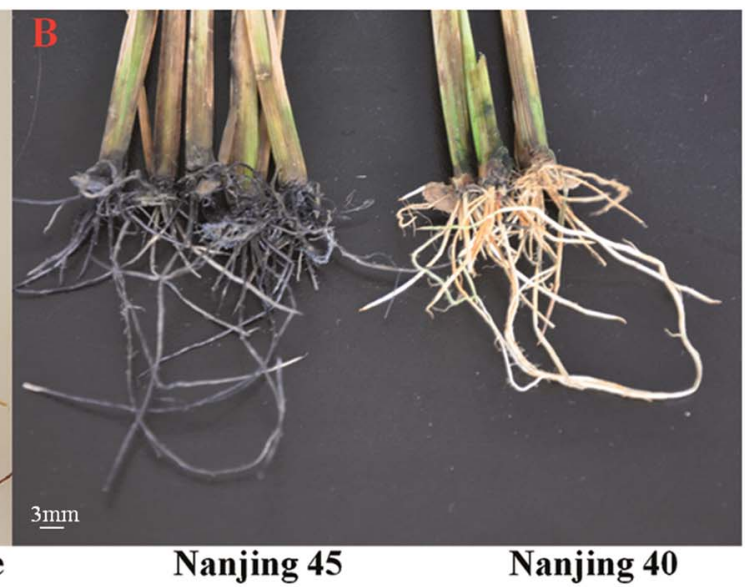

Nanjing 45
Nanjing 40

\footnotetext{
stem and root inoculation, respectively.
} 
deplete ribosomal RNA according to the manuscript of the RiboZero $^{\mathrm{TM}}$ rRNA Removal Kit, including four steps, prepare microspheres, treat sample with rRNA removal solution, remove rRNA and purify rRNA-depleted sample (Illumina, San Diego, USA). After removing ribosomal RNAs, the left RNAs were fragmented into small pieces using divalent cations under high temperature. Then the cleaved RNA fragments were reversetranscribed to create the cDNA. Single- or dual-index adapters are ligated to the fragments, and size (200-600 bp) selection was performed with agarose gel electrophoresis and the ligated products are amplified by PCR. At last, we performed the $100 \mathrm{bp}$ paired-end sequencing on an Illumina Hiseq 2500 following the recommended protocol.

\section{Identification of lncRNAs and analysis of sequencing data}

For identification of candidate lncRNAs, a computational pipeline was developed (Fig. 1). The raw data obtained from the two libraries were analyzed to remove adaptor, low-quality and rRNA reads. The remaining clean reads were aligned to the rice genome

(https://plants.ensembl.org/info/website/ftp/ index.html) using the TopHat2. ${ }^{23}$ Then, the mapped reads were utilized in transcript assembly by Cufflinks. According to the annotated rice transcripts (Automated Annotation of the Rice Genome), the known protein-encoding transcripts were removed, and unknown transcripts ( $>200$ and $<1500$ nucleotides) were defined as candidate lncRNA by using Cuffcompare. ${ }^{23}$ The remaining transcripts were aligned to miRBase to exclude miRNA precursors. Finally, novel lncRNAs were identified based on their non-protein coding potential (score $\leq-1$, means that the transcript has no potential probability for coding proteins) calculated by the Coding Potential Calculator (CPC; http://cpc.cbi.pku.edu.cn), and searching against the Pfam database using the software Hmmer to remove known non-coding RNAs. ${ }^{24,25}$

To measure the expression levels of lncRNAs and their targets, Cufflinks 2.0.0 was used to normalize counts of reads based on their lengths. Fragments Per Kilobase of exon model per Million mapped reads (FPKM) represented the normalized
A
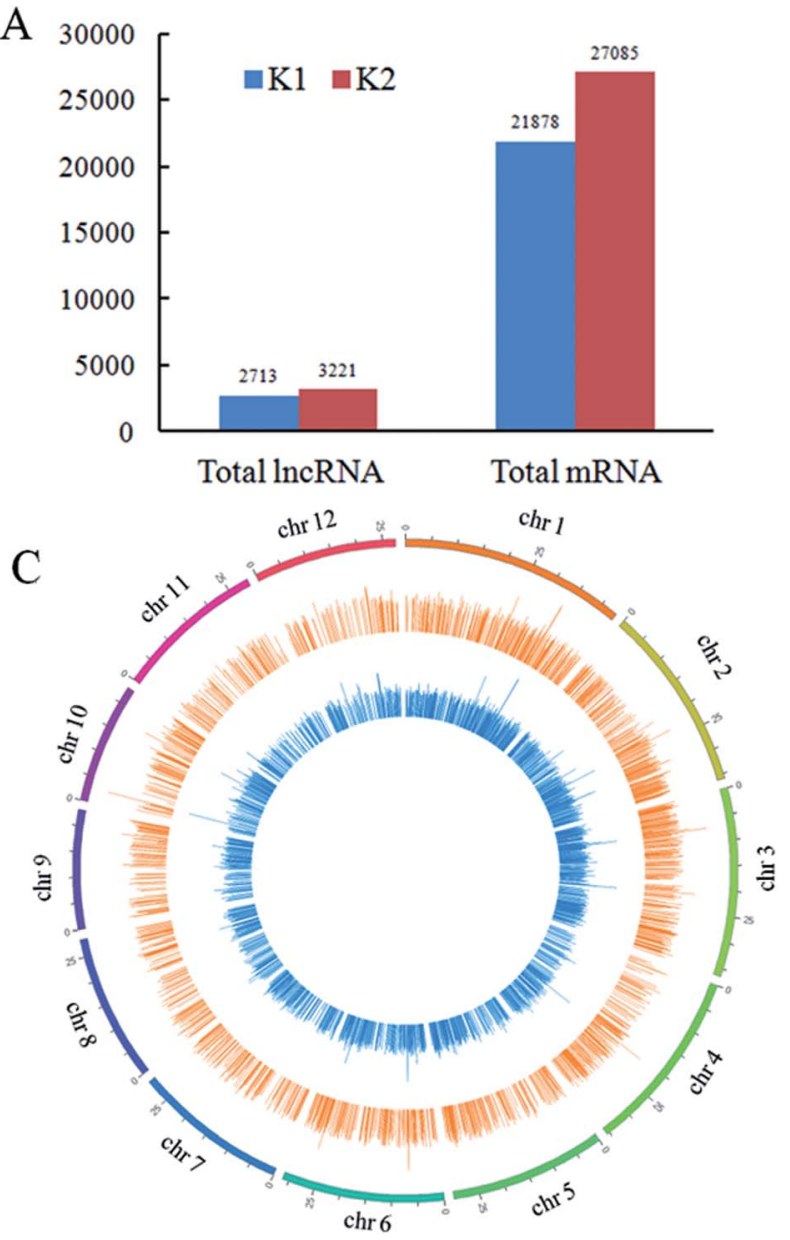

B
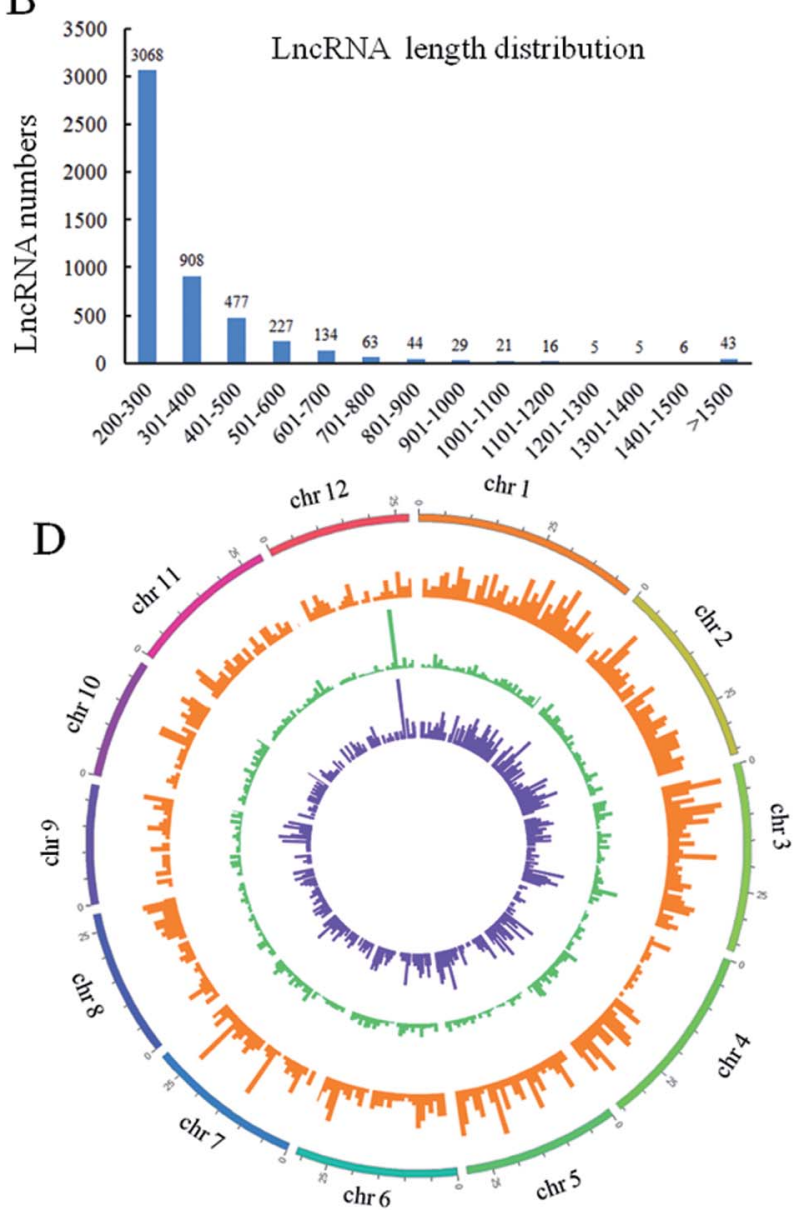

Fig. 3 Results of IncRNA identification. (A) The number of detected IncRNAs and mRNAs. (B) Length distribution of IncRNAs. (C) Genomic distribution of IncRNAs from two libraries. The outside concentric ring represents IncRNAs from K1, the inside concentric ring represents IncRNAs from K2. (D) Distribution of three kinds of IncRNAs. The numbers of intronic IncRNAs (outer orange circle), lincRNAs (middle green circle) and anti-sense IncRNAs (inner blue circle) in physical bins of $500 \mathrm{~kb}$ (kilobase) for each chromosome. Intronic IncRNAs are generated from the intron region of protein-coding gene. lincRNAs (long intergenic noncoding RNAs) are transcribed from intergenic regions of the rice genome. Antisense IncRNAs are transcribed from the antisense strand and overlap in part with one or more exons of a protein-coding gene. 
A

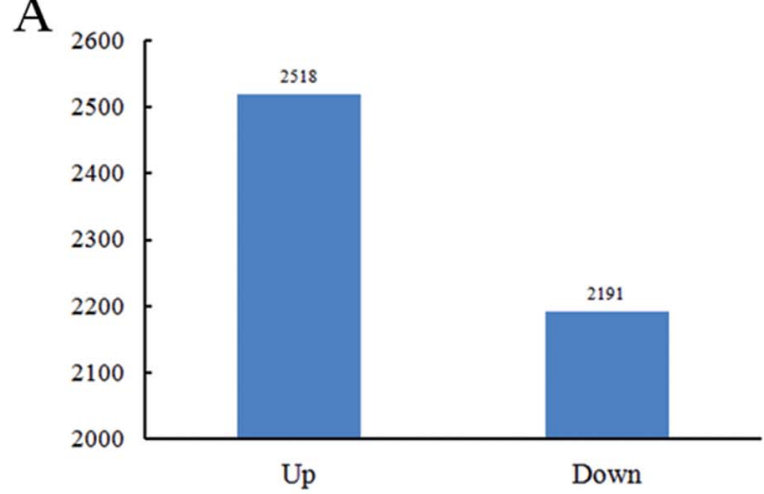

B

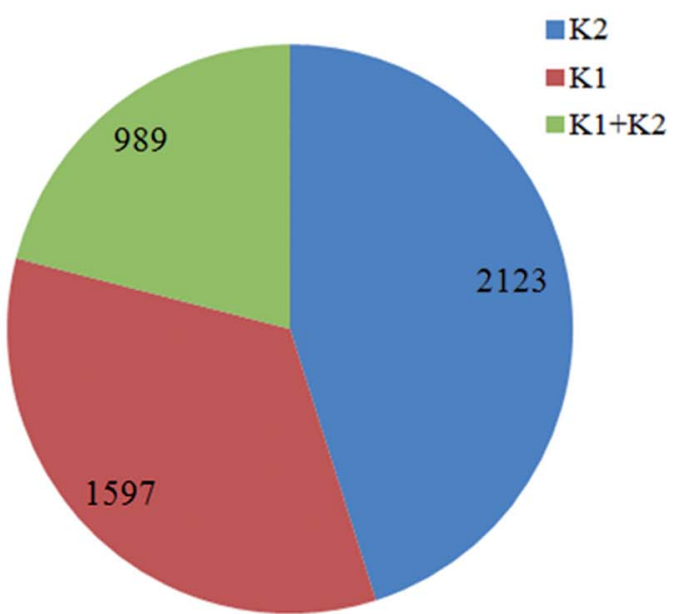

C
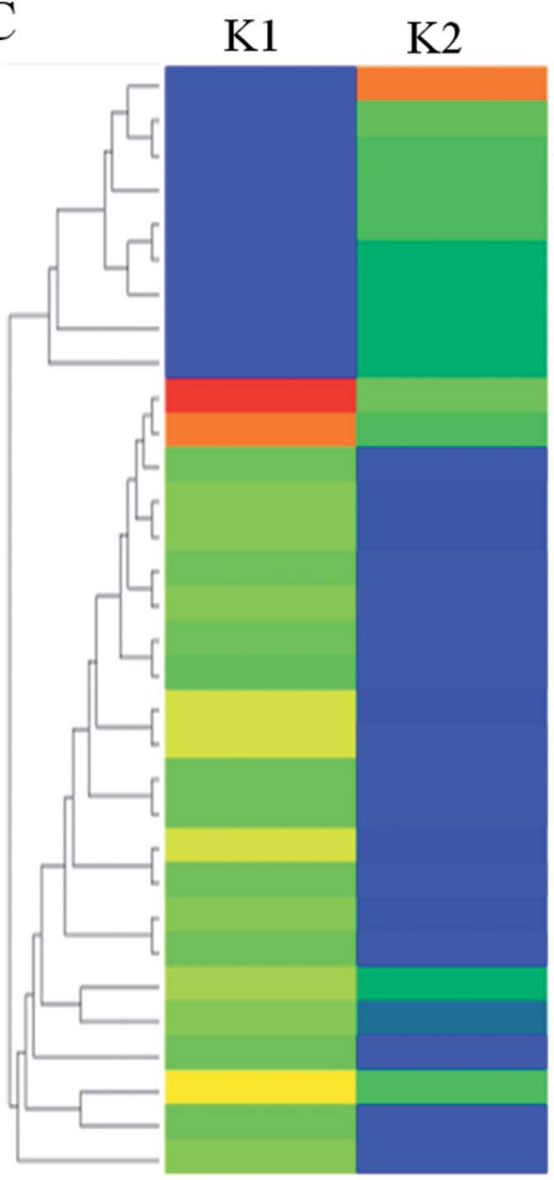

3284

3050

2815

2580

2346

2111

1877

1642

1407

1173

938

703

469

234

0.01

Fig. 4 Identification of IncRNAs responded to the infection of $D$. zeae in a resistant rice variety. (A) The number of differential expressed lncRNAs in a resistant rice variety after $D$. zeae inoculation. (B) The IncRNAs responded specifically to the infection by $D$. zeae in a resistant rice variety. K2 and $\mathrm{K} 1$ were the numbers of IncRNAs only detected in a resistant rice variety with and without inoculation. K1 + K2, the number of IncRNAs detected in both libraries. (C) Differentially expressed IncRNAs in two libraries by hierarchical clustering. More than 2 fold change was the cutoff point of the IncRNAs.

Table 1 The top ten up- and down-regulated targets in response to D. zeae infection based on RNA-seq

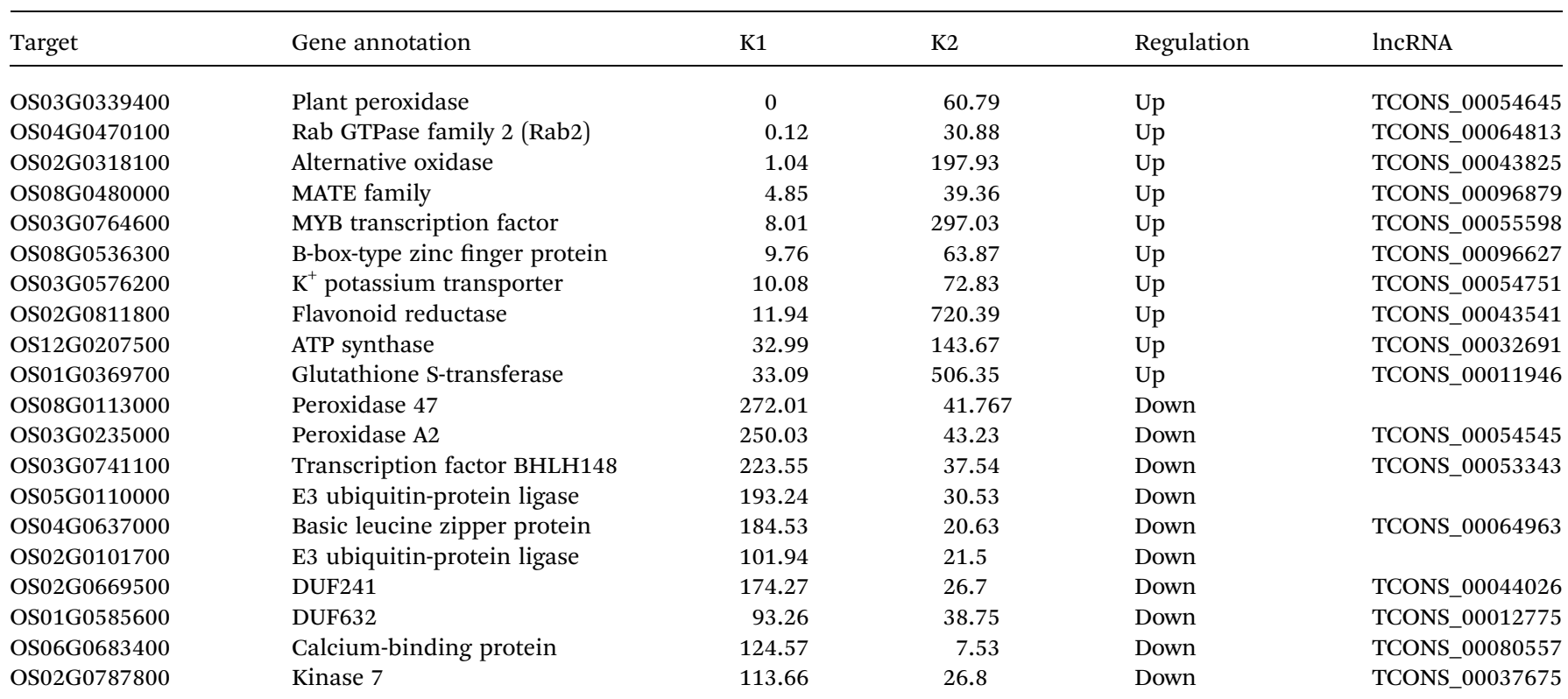


expression level of lncRNAs and target genes, and the significance of the expressional difference was tested by using $t$ test. The fold change was measured by the DEGseq package with $p$ values $<0.05$ and FDR (False Discovery Rate) $<0.05$, fold change $>2$ or $<-2$. The heatmap was developed according to the expression abundance of lncRNAs by using the software HemI (Heatmap Illustrator, version 1.0).

To analyze the cis regulation of IncRNAs, the Integrative Genomics Viewer was used to show the genomic loci containing IncRNAs and their adjacent genes. About $100 \mathrm{~kb}$ upstream or downstream sequences of IncRNAs were retrieved to search their candidate mRNA targets. Co-expressed lncRNA and target pairs were defined if both were expressed and spaced by less than $100 \mathrm{~kb}$. Furthermore, differentially expressed lncRNAs and mRNAs in response to D. zeae were identified.

\section{Validation of differentially expressed IncRNAs and targets}

To test the expression level of IncRNAs and their predicted targets, rice roots after inoculation were collected for extraction of total RNA from two samples, K1 (0 hpi) and K2, a mixed sample of 6, 12 and 48 hpi using the Trizol reagent (TaKaRa,
Dalian, China). Quantitative PCR (qPCR) analysis was done using Applied Biosystem 7500 Real Time PCR System and SYBR Premix Ex Taq ${ }^{\mathrm{TM}}$ (TaKaRa, Dalian, China) according to the manufacturer's instructions. The first-strand cDNA was synthesized by the First-strand cDNA Synthesis Kit (TaKaRa, Dalian, China) using random hexamer primer (lncRNAs) or oligo dT RTprimer (mRNAs). The EF1- $a$ gene was used as the reference control gene to standardize the RNA sample for evaluating relative expression levels, and all primers used in this study were listed in Table S1. $\dagger$ For qPCR assays, three independent biological samples were carried out, accompanied by three technical replicates.

\section{Results}

\section{Response of a resistant japonica rice to $D$. zeae infection}

Hypersensitive response inhibiting the infection and invasion of $D$. zeae in a resistant rice variety Nanjing 40 was observed whereas three susceptible rice varieties, Nanjing 45, Kasalath and Nipponbare showed typical disease symptoms nearby the site of injection at $10 \mathrm{dpi}$ (Fig. 2A). Moreover, according to the

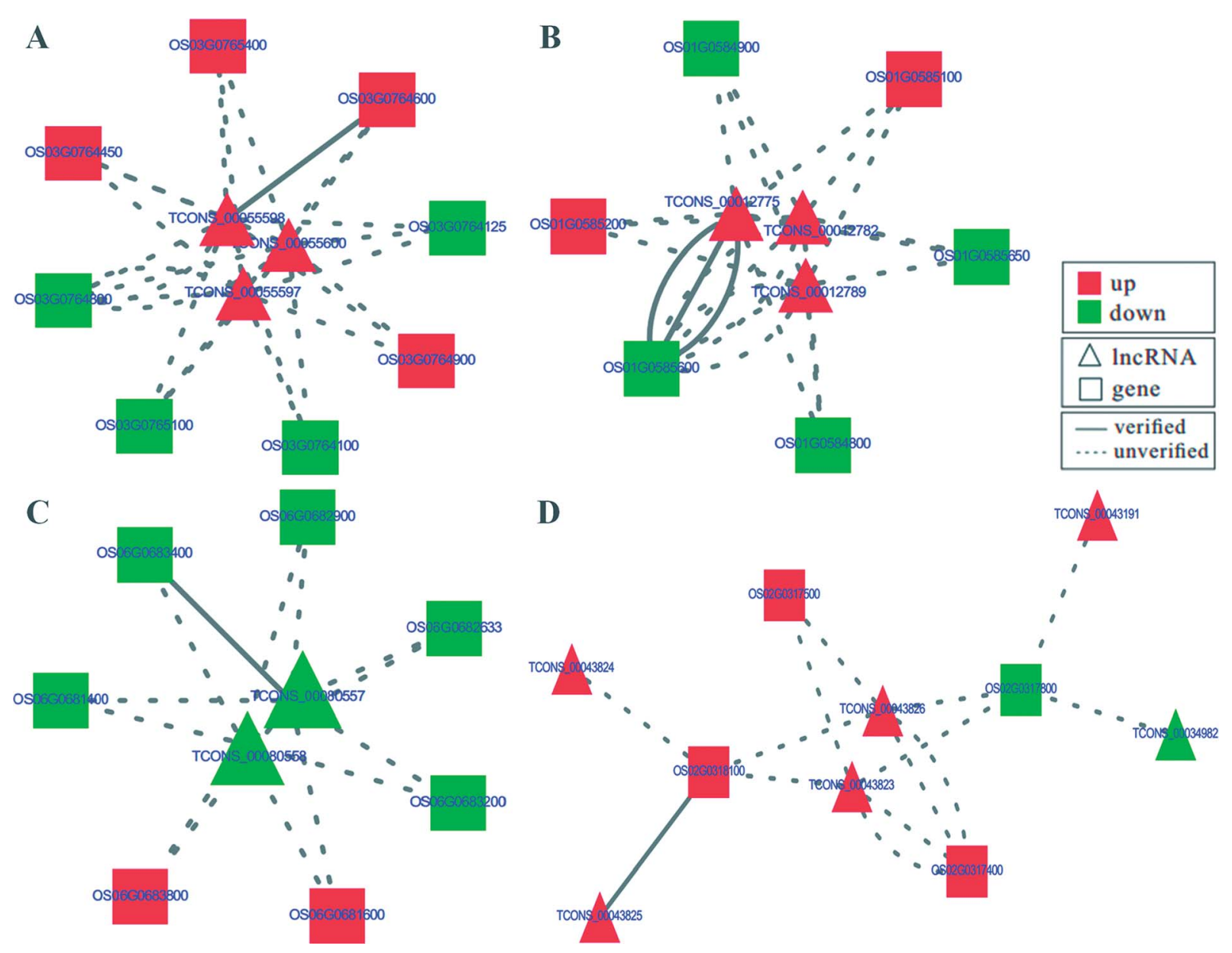

Fig. 5 The predicted interaction networks of four IncRNAs-target pairs. (A)-(D) show the interaction networks of different lncRNA-target pairs. The triangular and rectangular nodes represent IncRNAs and relevant targets, respectively. Red and green represent the up-regulated and downregulated nodes, respectively. Lines show the interaction of the IncRNAs-target pairs. The solid line and break line represent verified and unverified IncRNA-target pairs, respectively in Table 1. 
Table 2 Expression profile of IncRNAs acting as ceRNAs in response to $D$. zeae infection in resistant rice

\begin{tabular}{|c|c|c|c|c|c|}
\hline \multirow[b]{2}{*}{ miR_name } & \multirow[b]{2}{*}{ ceRNA } & \multirow[b]{2}{*}{ Predicted target } & \multirow[b]{2}{*}{ Gene annotation } & \multicolumn{2}{|c|}{ ceRNA } \\
\hline & & & & K1 & $\mathrm{K} 2$ \\
\hline osa-miR156 & TCONS_00043299 & OS02G0557300 & RING-H2 finger protein & 0 & 81 \\
\hline osa-miR156 & TCONS_00102384 & OS09G0376800 & Glycosyltransferase & 1980 & 778 \\
\hline osa-miR156 & TCONS_00102574 & OS09G0252800 & Ubiquitin-protein ligase & 267 & 163 \\
\hline osa-miR396 & TCONS_00042166 & OS02G0742100 & DNA binding protein & 1096 & 481 \\
\hline osa-miR396 & TCONS_00064123 & OS04G0112200 & Transcription factor E2FB & 0 & 134 \\
\hline osa-miR396 & TCONS_00081640 & OS06G0503400 & Reticulon-like protein B2 & 0 & 193 \\
\hline
\end{tabular}

root inoculation experiment, Nanjing 40 was also more resistant to $D$. zeae than Nanjing 45 , which showed withered leaves, black rot symptom and foul smell (Fig. 2B). The results from both two inoculation experiments demonstrated that Nanjing 40 was highly resistance against $D$. zeae and was thus chosen to analyze resistance mechanism mediated by lncRNA against $D$. zeae using the high-through sequencing technology.

\section{Overview of RNA-seq in an incompatible interaction of rice with $D$. zeae}

In order to understand the molecular mechanisms of rice lncRNAs responsive to D. zeae in resistant rice, the raw reads from RNA-seq data were analyzed respectively, and the raw reads were submitted to NCBI (SRA: SRP149059). 4841 lncRNAs in Nanjing 40 were identified. Among them, 3221 and 2713 transcripts were identified in the infected (K2) and control (K1) sample, respectively (Fig. 3A and Table S2 $\dagger$ ). The lengths of the detected lncRNAs were in the range from 200 to more than 1500 bp, and 200-300 bp lncRNAs accounted for 60.8\% (Fig. 3B). These lncRNAs were evenly distributed on 12 rice chromosomes (Fig. 3C). According to their locations in the genome, 1581, 2138 and 1122 were classified as intronic lncRNAs, lincRNAs (also termed long intergenic noncoding RNAs or intergenic lncRNAs)

Table 3 The 17 differentially expressed IncRNAs based on the normalized data and randomly chosen from RNA-seq data

\begin{tabular}{lccc}
\hline lncRNA & K1 & K2 & Regulation \\
\hline TCONS_00043825 & 120.87 & 980.18 & Up \\
TCONS_00032691 & 0 & 168.89 & Up \\
TCONS_00064597 & 0 & 139.36 & Up \\
TCONS_00054751 & 0 & 845.71 & Up \\
TCONS_00096627 & 0 & 104.63 & Up \\
TCONS_00054645 & 0 & 138.92 & Up \\
TCONS_00054417 & 0 & 97.72 & Up \\
TCONS_00096879 & 0 & 142.38 & Up \\
TCONS_00081726 & 0 & 147.87 & Up \\
TCONS_00064813 & 0 & 99.03 & Up \\
TCONS_00064963 & 0 & 83.99 & Up \\
TCONS_00044026 & 0 & 63.73 & Up \\
TCONS_00012775 & 0 & 77.49 & Up \\
TCONS_00054545 & 1621.13 & 195.11 & Down \\
TCONS_00053343 & 1020.79 & 17.03 & Down \\
TCONS_00037675 & 274.84 & 0 & Down \\
TCONS_00080557 & 620.79 & 0 & Down
\end{tabular}

and antisense lncRNAs. Their distribution on the 12 chromosomes are shown by different concentric rings from outside to inside in Fig. 3D (Fig. S1†).

\section{Identification of $D$. zeae responsive IncRNAs}

A total of 4709 lncRNAs in response to the infection of D. zeae were identified $(P$-value $<0.05)$ in roots of K1 sample compared with K2 sample, including 2518 up-regulated lncRNAs and 2191 down-regulated IncRNAs (Fig. 4A, S2 and Table S3†). Additionally, 2123 lncRNAs were uniquely detected in Nanjing 40 after $D$. zeae inoculation, 1597 lncRNAs were unique to the control, and 989 lncRNAs were detected in both libraries (Fig. 4B and Table $\mathrm{S} 3 \dagger)$. The heatmap in Fig. 4C showed the selected 33 IncRNAs differentially expressed in an incompatible interaction (Fig. 4C and Table $\mathrm{S} 4 \dagger$ ). Differential expression of lncRNAs at certain time points after $D$. zeae infection suggests a role of these lncRNAs in response to $D$. zeae infection.

\section{Co-expression analysis between IncRNAs and their targets in defense pathways}

To identify potential mRNA targets of lncRNAs, differential expression was analyzed for the mRNAs within the $100 \mathrm{~kb}$ flanking regions of differentially expressed lncRNAs. ${ }^{26}$ The transcriptome data from RNA-seq identified top ten upregulated targets involved in multiple signal pathways related to defense responses, such as plant peroxidase, Rab GTPase family 2 (Rab2), alternative oxidase, MATE family protein, MYB family transcription factor, $\mathrm{K}^{+}$potassium transporter, flavonoid reductase and glutathione $S$-transferase etc. (Table 1). The top ten down-regulated targets included peroxidase 47, peroxidase A2, two E3 ubiquitin-protein ligases, two DUFs (Table 1). Most of these targets, including peroxidase and E3 ubiquitin-protein ligases, are involved in defense response signal pathways. ${ }^{27} \mathrm{Co}^{-}$ expression analysis of lncRNAs and their flanking mRNAs uncovered that 17 lncRNAs regulated the expression of 17 of 20 targets, except for OS08G0113000, OS05G0110000 and OS02G0101700 (Table 1). Moreover, the interactive networks of 17 IncRNA-target pairs were constructed by the Cytoscape software. Seven of them had networks with more than four nodes, and the remaining ten had networks with three or less nodes (Fig. 5 and S3†). The interactive networks shown in Fig. 5A contained eight target genes, including MYB 
A
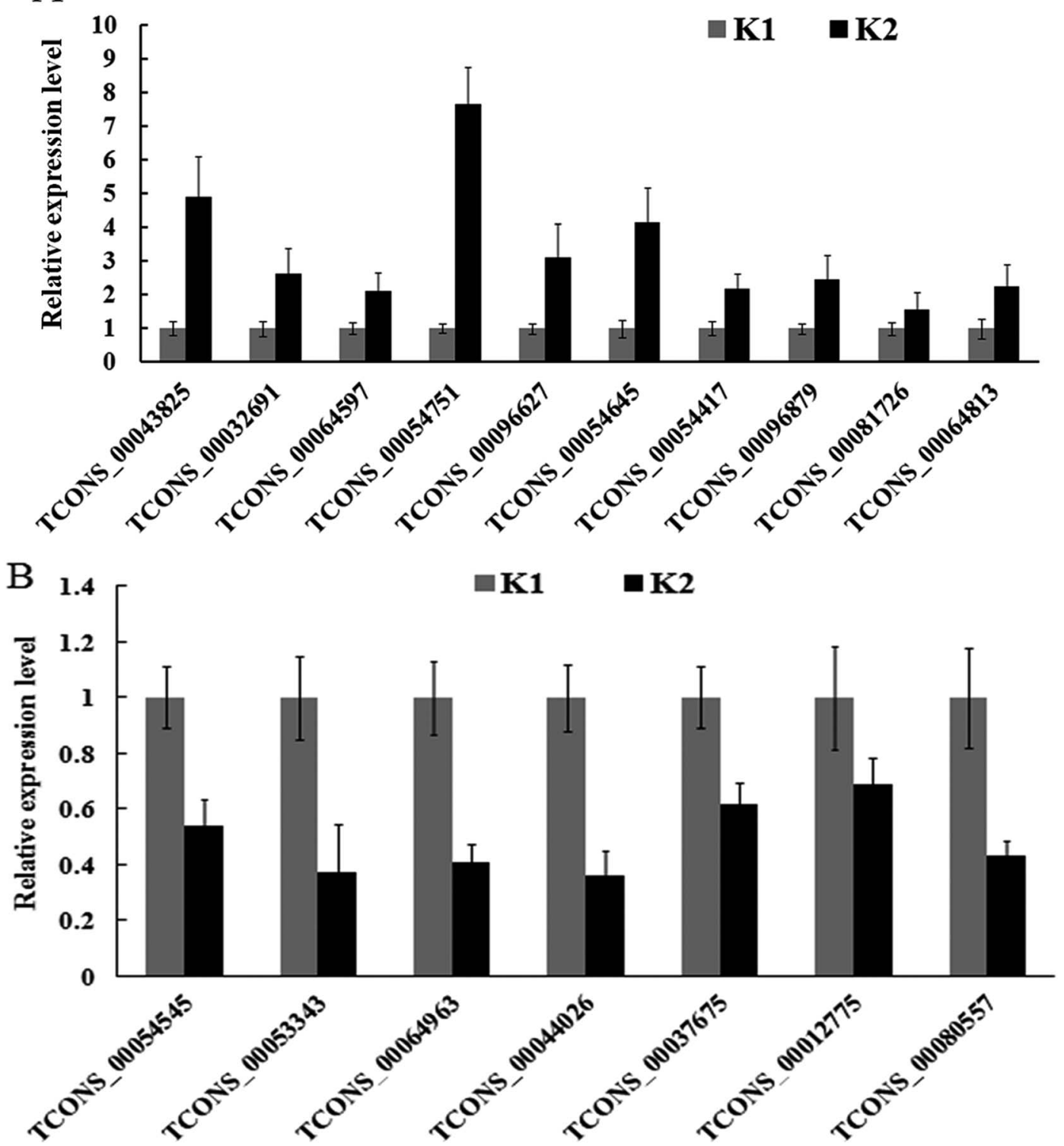

Fig. 6 IncRNAs from RNA-seq verified by qPCR. (A) and (B) the expression level of ten up-regulated and seven down-regulated lncRNAs were validated by QPCR, respectively. Data represent means of three replicates \pm standard deviation for each target in the two libraries.

transcription factor, zinc finger and serine/threonine-protein kinase, which were regulated by three IncRNAs (TCONS_00055600, TCONS_00055598 and TCONS_00055597) in the resistant rice cultivar in response to $D$. zeae infection. The interactive network shown in Fig. 5B represented three lncRNAs (TCONS_00012775, TCONS_00012782 and TCONS_00012789) regulating six targets, including gene encoding component of the small subunit processome, protein with domains of unknown function (DUFs) and WRKY transcription factor. The targets of TCONS_00080557 and TCONS_00080558 were calcium binding motif-containing protein, phospho-3sulfolactate synthase, $50 \mathrm{~S}$ ribosomal protein L24 and peroxidase (Fig. 5C). Moreover, a more complex interactive network was found between six IncRNAs and four targets (Fig. 5D). These four targets were composed of uncharacterized protein (OS02G0317800), alternative oxidase (OS02G0318100), F-box- like protein with seven leucine-rich repeats (LRR; OS02G0317500) and clathrin adaptor (OS02G0317400), which OS02G0318100 and OS02G0317500 may respond to the infection of $D$. zeae in rice. The remaining 13 interactive networks of IncRNAs-target pairs were shown in Fig. S3. $\uparrow$ Targets involved in these networks included plant peroxidase, alternative oxidase, peroxidase, transcription factor, E3 ubiquitin-protein ligase and so on. These lncRNAs-target pairs may have a regulatory role in defense against $D$. zeae infection.

\section{Crosstalk of the IncRNA-miRNA network in an incompatible interaction}

Previously, two miRNAs, osa-miR396 and osa-miR156, have been shown to be involved in the rice defense responses respectively. ${ }^{28,29}$ The IncRNAs acting as potential ceRNAs 

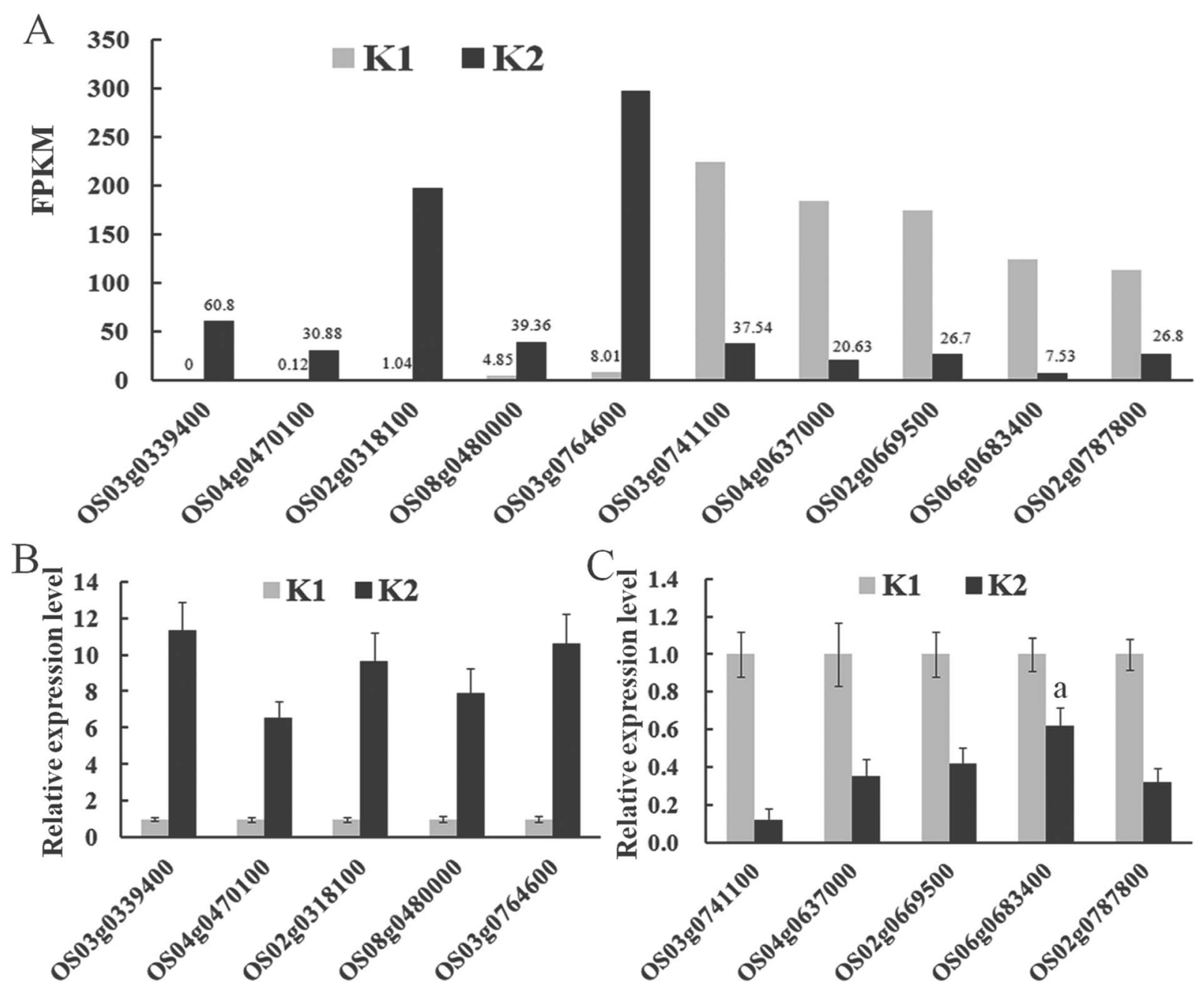

Fig. 7 Expression levels of the ten targets selected for verification by qPCR. (A) Ten significantly differentially expressed target genes based on the number of normalized reads in an incompatible interaction. FPKM (Fragments Per Kilobase of exon model per Million mapped reads) represents the normalized expression level of target genes. (B) and (C) The expression level of five up- and down-regulated targets analyzed by qPCR, respectively. Data represent means of three replicates \pm standard deviation for each target in the two libraries in an incompatible interaction. a indicates that a significant difference $(P<0.05)$ was detected between K1 and K2.

mimics of both miR396 and miR156 were predicted and their expressional changes were analyzed using the lncRNA sequencing data (Table 2). Three IncRNAs, TCONS_00042166, TCONS_00064123 and TCONS_00081640, were predicted to be ceRNAs mimics of osa-miR396 (Table 2). Six IncRNAs, TCONS_00012844, TCONS_00043299, TCONS_00102384, TCONS_00102574, TCONS_00072934 and TCONS_00013133, were predicted ceRNAs of osa-miR156 (Table 2). It is noticeable, some mRNA genes were presumably the targets of miRNAs osamiR156 and osa-miR396 (Table 2), predicted to be as ubiquitinprotein ligase, vacuolar-sorting receptor protein, esterase protein and transcription factors involved in defense responses (Table 2) suggesting that IncRNA may regulate miRNAs in resisting $D$. zeae in an incompatible interaction.

\section{Validation of $D$. zeae responsive lncRNAs}

A total of 17 differentially expressed lncRNAs were randomly chosen for qPCR analysis. Among the 17 lncRNAs, 13 had their number of normalized reads significantly up-regulated, and four significantly down-regulated (Table 3 ). For example, the number of normalized reads of IncRNA TCONS_00054751 in the infected and control samples were 845.71 and 0 , respectively (Table 3), representing the highest induced IncRNA. The qPCR analysis results confirmed the expression changes of 17 IncRNAs, although three IncRNAs (TCONS_00064963, TCONS_00044026 and TCONS_00012775) showed an opposite result (Fig. 6).

Ten targets (five up-regulated and five down-regulated genes in response to $D$. zeae infection) were randomly selected for confirmation of their differential expression pattern using qPCR (Fig. 7). For all ten genes, their expression patterns were confirmed despite the degree of changes varied between RNAseq (Fig. 7A) and qPCR (Fig. 7B and C).

\section{Discussion}

Rice foot rot disease caused by pathogen $D$. zeae is one of the most destructive bacterial diseases. ${ }^{1}$ IncRNAs were involved in 
the regulation of target gene expression at epigenetic, transcriptional and post-transcriptional levels in rice. ${ }^{16}$ It was predicted that lncRNA plays a role in a wide range of biological processes including growth and development and stress responses in plants. ${ }^{\mathbf{1 4 1 5}}$ In this study RNA-seq was performed to identify the putative rice lncRNAs in response to $D$. zeae infection in Nanjing 40.

lncRNAs are thought to be involved in complex interaction with target genes and miRNAs. ${ }^{19}$ IncRNAs may function as ceRNA to bind specific miRNAs to prevent degradation of relevant target mRNAs by miRNAs. ${ }^{18}$ To a great degree, the biological function of miRNAs partly reflected the biological function of lncRNAs as ceRNAs. ${ }^{19}$ The Plant ceRNA database (PceRBase, http://bis.zju.edu.cn/pcernadb/index.jsp) contains potential ceRNAs, and ceRNA target-mimic pairs from 26 plant species. ${ }^{17}$ lncRNAs potentially acting as ceRNAs of two key miRNAs, osamiR156 and osa-miR396, were deduced and characterized in this study (Table 2). These two miRNAs regulate not only growth and development but also response to pathogen infection in plants. ${ }^{29}$ Particularly, a recent study showed that rice blast infection significantly induced the accumulation of osa-miR156 and osa-miR396. ${ }^{29}$ In this study, we identified that ubiquitinprotein ligase and glycosyltransferase are two targets of osamiR156 (Table 2). Previous study demonstrated that ubiquitin ligase-associated protein SGT1 is required for resistance responses to diverse phytopathogens in Nicotiana benthamiana. ${ }^{30}$ Recent study showed that E3 ubiquitin ligase EBR1 interacted with target OsBAG4 in triggering programmed cell death and activating innate immunity and resistance to both blast and Xoo in rice. ${ }^{27}$ Moreover, glycosyltransferase plays a key role in regulating the composition of plant cell wall resulted in increased diseases resistance. ${ }^{31,32}$ Overexpression of miR396a or miR396b in Arabidopsis significantly contributed resistance to cyst nematode. ${ }^{31}$ We also identified that reticulon-like protein B2 and transcription factor E2FB-like were the predicted two targets of osa-miR396 (Table 2). The reticulon-like protein B2 gene was clearly induced by the fungal pathogen Fusarium oxysporum in chickpea. ${ }^{33}$ The E2FB transcription factor in Arabidopsis may be involved in the bacterial disease resistance by regulating the auxin signal pathway. ${ }^{33,34}$ Another predicted target of osa-miR396 was the growth-regulate factor $O s G R F$, which is involved in multiple resistance-related signal pathways such as cell-wall modification, cytokinin biosynthesis and the accumulation of secondary metabolites in Arabidopsis. ${ }^{31,32}$

The reactive oxygen species (ROS) burst was a key defense responses signal molecule conferring the broad-spectrum resistance to fungal and bacterial diseases in rice. ${ }^{35}$ For example, rapid accumulation of ROS resulting from the inhibition of the expression of peroxidase gene in $b s r-d 1$ knockout plants conferred a broad-spectrum resistance to rice blast fungus. ${ }^{35}$ We showed that the expression levels of the targets of TCONS_00055598 (OS03G0764600, a MYB transcription factor) and TCONS_00054545 (OS03G0235000, peroxidase A2), were significantly up- and down-regulated, respectively (Table 1 and Fig. 7). In addition, plant peroxidase (OS03G0339400) and alternative oxidase (OS02G0318100), targets of TCONS_00054645 and TCONS_00043825, respectively, were increased in the incompatible interaction (Table 1 and Fig. 7). During an incompatible interaction, increase of peroxidase suggests that lncRNAs may regulate ROS during defense against D. zeae. Consistent with the observation that ROS balance mechanism can be used to prevent the damage resulting from massive ROS burst induced by pathogen infection. ${ }^{36}$

In this study, we identified and characterized lncRNAs in response to $D$. zeae infection in a resistant rice variety using RNA-seq. A total of 4709 lncRNAs, 2518 up-regulated and 2191 down-regulated, were detected. The RNA-seq based expression levels of selected IncRNAs and their targets were validated by qPCR. Some lncRNAs were predicted to be target mimics of miRNAs. These results in this study provide evidences for better understanding the role of lncRNAs in regulating resistance to the bacterial rice foot rot disease in rice, as well as show a potential approach for cultivating the resistant rice to foot rot disease by generating lncRNA transgenic plants in the future.

\section{Conflicts of interest}

All authors declare that there are no conflicts of interest.

\section{Acknowledgements}

We thank Dr Lei Liu of Genepioneer Biotechnologies for his help of bioinformatics analysis. This work was supported by the National Key Research and Development Program (No. 2017YFD0100400-3), the Jiangsu Key Laboratory of Agrobiology Program (No. 49114042015Z006), the Jiangsu Province Key Research and Development Program (Modern Agriculture) Project (No. BE2015355), the Exploratory Project of the Jiangsu Academy of Agricultural Sciences (No. ZX(17)2014), and the Jiangsu Province Natural Science Foundation (No. BK20171326). The United States Department of Agriculture (USDA) is an equal opportunity provider and employer.

\section{References}

1 Q. G. Liu, Q. Zhang and C. D. Wei, Sci. Agric. Sin., 2013, 46, 2923-2931.

2 M. Goto, Phytopathology, 1979, 69, 213-216.

3 J. N. Zhou, H. B. Zhang, J. Wu, Q. G. Liu, P. G. Xi, J. Lee, J. L. Liao, Z. D. Jiang and L. H. Zhang, Mol. Plant-Microbe Interact., 2011, 24, 1156-1164.

4 J. Y. Liu, J. H. Rice, N. N. Chen, T. J. Baum and T. Hewezi, PLoS One, 2014b, 9, e98477.

5 L. Yang and H. Huang, J. Integr. Plant Biol., 2014, 56, 962970.

6 J. A. Chekanova, Curr. Opin. Plant Biol., 2015, 27, 207-216.

7 X. Liu, L. L. Hao, D. Y. Li, L. H. Zhu and S. N. Hu, Genomics, Proteomics Bioinf., 2015, 13, 137-147.

8 H. Wang, Q. W. Niu, H. W. Wu, J. Liu, J. Ye, N. Yu and N. H. Chua, Plant J., 2015, 84, 404-416.

9 J. Mach, Plant Cell, 2017, 29, 916.

10 Q. H. Zhu, S. Stephen, J. Taylor, C. A. Helliwell and M. B. Wang, New Phytol., 2014, 201, 574-584. 
11 H. Zhang, W. G. Hu, J. L. Hao, S. K. Lv, C. Y. Wang, W. Tong, Y. J. Wang, Y. Z. Wang, X. L. Liu and W. Q. Ji, BMC Genomics, 2016, 17, 238.

12 P. J. Chung, H. Jung, D. H. Jeong, S. H. Ha, Y. D. Choi and J. K. Kim, BMC Genomics, 2016, 17, 563.

13 D. D. Niu, X. Zhang, X. O. Song, Z. H. Wang, Y. Q. Li, L. L. Qiao, Z. Y. Wang, J. Z. Liu, Y. W. Deng, Z. H. He, D. L. Yang, R. Y. Liu, Y. L. Wang and H. W. Zhao, Phytopathology, 2018, 108, 60-69.

14 J. H. Ding, Q. Lu, Y. D. Ouyang, H. L. Mao, P. B. Zhang, J. L. Yao, C. G. Xu, X. H. Li, J. H. Xiao and Q. F. Zhang, Proc. Natl. Acad. Sci. U. S. A., 2012, 109, 2654-2659.

15 Y. C. Zhang, J. Y. Liao, Z. Y. Li, Y. Yu, J. P. Zhang, Q. F. Li, L. H. Qu, W. S. Shu and Y. Q. Chen, Genome Biol., 2014, 15, 512 .

16 Q. H. Zhu and M. B. Wang, Genes, 2012, 3, 176-190.

17 C. H. Yuan, X. W. Meng, X. Li, N. Illing, A. R. Ingle, J. J. Wang and M. Chen, Nucleic Acids Res., 2017, 45(D1), D1009-D1014.

18 H. J. Wu, Z. M. Wang, M. Wang and X. J. Wang, Plant Physiol., 2013, 161, 1875-1884.

19 Y. Tay, J. Rinn and P. P. Pando, Nature, 2014, 505, 344-352.

20 X. W. Xu, X. H. Zhou, R. R. Wang, W. L. Peng, Y. An and L. L. Chen, Sci. Rep., 2016, 6, 20715.

21 W. Q. Li, J. Yang, J. Wang, F. F. Fan, J. Y. Zhu, F. Q. Wang and W. G. Zhong, Jiangsu Agricultural Sciences, 2014, 42, 139-142.

22 B. A. Besma, W. Sonia, M. Francisco, L. Philippe, d'A. C. Yves, H. Judith, M. Alexis, M. Allison, L. Antoine, M. D. Jean, V. Herve, T. Claude and C. Martin, Genome Res., 2009, 19, 57-69.

23 D. Kim, G. Pertea, C. Trapnell, H. Pimentel, R. Kelley and S. L. Salzberg, Genome Biol., 2013, 14, R36.

24 L. Kong, Y. Zhang, Z. Q. Ye, X. Q. Liu, S. Q. Zhao, L. P. Wei and G. Gao, Nucleic Acids Res., 2007, 35, W345-W349.

25 Y. N. Pang, C. B. Mao and S. R. Liu, Theranostics, 2018, 8, 2496-2507.

26 X. D. Hou, Y. M. Du, X. M. Liu, H. B. Zhang, Y. H. Liu, N. Yan and Z. F. Zhang, Int. J. Mol. Sci., 2008, 19, 101.
27 Q. Y. You, K. R. Zhai, D. L. Yang, W. B. Yang, J. N. Wu, J. Z. Liu, W. B. Pan, J. J. Wang, X. D. Zhu, Y. K. Jian, J. Y. Liu, Y. Y. Zhang, Y. W. Deng, Q. Li, Y. G. Lou, Q. Xie and Z. H. He, Cell Host Microbe, 2016, 20, 758-769.

28 T. Hewezi, T. R. Maier, D. Nettleton and T. J. Baum, Plant Physiol., 2012, 159, 321-335.

29 Z. Y. Li, J. Xia, Z. Chen, Y. Yu, Q. F. Li, Y. C. Zhang, J. P. Zhang, C. Y. Wang, X. Y. Zhu, W. Zhang and Y. Q. Chen, Sci. Rep., 2016, 6, 25493.

30 J. R. Peart, R. Lu, A. Sadanandom, I. Malcuit, P. Moffett, D. C. Brice, L. Schauser, D. A. W. Jaggard, S. Y. Xiao, M. J. Coleman, M. Dow, J. D. G. Jones, K. Shirasu and D. C. Baulcombe, Proc. Natl. Acad. Sci. U. S. A., 2002, 99, 10865-10869.

31 C. Hernández-Blanco, D. X. Fen, J. Hu, A. Sánchez-Vallet, L. Deslandes, F. Llorente, M. Berrocal-Lobo, H. Keller, X. Barlet, C. Sánchez-Rodríguez, L. K. Anderson, S. Somerville, Y. Marco and A. Molina, Plant Cell, 2007, 19, 890-903.

32 H. J. Park, C. S. Kwon, J. Y. Woo, G. J. Lee, Y. J. Kim and K. H. Paek, Plant Pathol. J., 2011, 27, 170-182.

33 M. L. Upasani, B. M. Limaye, G. S. Gurjar, S. M. Kasibhatla, R. R. Joshi, N. Y. Kadoo and V. S. Gupta, Sci. Rep., 2017, 7, 7746.

34 Z. Magyar, L. D. Veylder, A. Atanassova, L. Bakó, D. Inzé and L. Bögre, Plant Cell, 2005, 17, 2527-2541.

35 W. T. Li, Z. W. Zhu, M. S. Chern, J. J. Yin, C. Yang, L. Ran, M. P. Cheng, M. He, K. Wang, J. Wang, X. G. Zhou, X. B. Zhu, Z. X. Chen, J. C. Wang, W. Zhao, B. T. Ma, P. Qin, W. L. Chen, Y. P. Wang, J. L. Liu, W. M. Wang, X. J. Wu, P. Li, J. R. Wang, L. H. Zhu, S. G. Li and X. W. Chen, Cell, 2017, 170, 114-126.

36 W. Q. Li, M. Shao, W. G. Zhong, J. Yang, K. Okada, H. Yamane, L. Zhang, G. Wang, D. Wang, S. S. Xiao, S. S. Chang, G. L. Qian and F. Q. Liu, PLoS One, 2012, 7, e43914. 